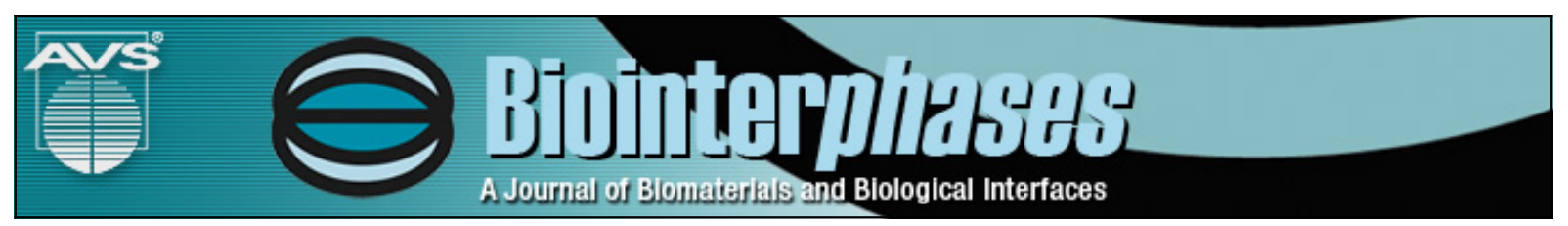

\title{
Laser surface structuring of AZ31 Mg alloy for controlled wettability
}

Ali Gökhan Demir, Valentina Furlan, Nora Lecis, and Barbara Previtali

Citation: Biointerphases 9, 029009 (2014); doi: 10.1116/1.4868240

View online: http://dx.doi.org/10.1116/1.4868240

View Table of Contents: http://scitation.aip.org/content/avs/journal/bip/9/2?ver=pdfcov

Published by the AVS: Science \& Technology of Materials, Interfaces, and Processing 


\title{
Laser surface structuring of AZ31 Mg alloy for controlled wettability
}

\author{
Ali Gökhan Demir, ${ }^{\text {a) }}$ Valentina Furlan, ${ }^{\text {b) }}$ Nora Lecis, ${ }^{\text {c) }}$ and Barbara Previtali ${ }^{\text {d) }}$ \\ Department of Mechanical Engineering, Politecnico di Milano, Via La Masa 1, 20156 Milan, Italy
}

(Received 15 January 2014; accepted 28 February 2014; published 14 March 2014)

\begin{abstract}
Structured surfaces exhibit functional properties that can enhance the performance of a bioimplant in terms of biocompatibility, adhesion, or corrosion behavior. In order to tailor the surface property, chemical and physical methods can be used in a sequence of many steps. On the other hand, laser surface processing can provide a single step solution to achieve the designated surface function with the use of simpler equipment and high repeatability. This work provides the details on the surface structuring of AZ31, a biocompatible and biodegradable Mg alloy, by a single-step laser surface structuring based on remelting. The surfaces are characterized in terms of topography, chemistry, and physical integrity, as well as the effective change in the surface wetting behavior is demonstrated. The results imply a great potential in local or complete surface structuring of medical implants for functionalization by the flexible positioning of the laser beam. (C) 2014 American Vacuum Society. [http://dx.doi.org/10.1116/1.4868240]
\end{abstract}

\section{INTRODUCTION}

Magnesium alloys are gaining more attention from both research and industrial communities in biomedical implant manufacturing. Due to high biocompatibility and biodegradability properties, $\mathrm{Mg}$ alloys are suitable implant materials for wires, pins, and nails as orthopedic implants and in treatment of cardiovascular diseases. ${ }^{1,2}$ More recently, their use as biodegradable stent material has been demonstrated. ${ }^{3}$ However, Mg alloys are also characterized by low corrosion resistance to body fluids due to the high biocompatibility. Especially in the case of biodegradable stent applications, the degradation rate of the $\mathrm{Mg}$ alloy stent may be lower than the required period of 6 months for vessel remodeling. ${ }^{4}$ The surface characteristics, which mainly consist of surface topography, chemistry, and wettability, play a crucial role on how the implant material interacts with the surrounding media. These interactions also involve corrosion rate and mechanism as well as biological response of the tissue to the implant material. If an intermediate layer such as a coating or drug should be added to the implant, the same surface characteristics determine the efficacy of the adherence of the intermediate layer. In particular, high wettability is a desired property to improve adhesion of surface coatings.

From this perspective, the use of a laser beam allows many possibilities in terms of controlling surface characteristics. This technology does not require operating in vacuum unlike other energetic beam technologies (i.e., ion beam, electron beam) and is considered as a sterile tool as there is no direct contact with the material being processed. ${ }^{5}$ Today's beam manipulation systems allow large area processing using masked projection techniques as well as localized surface processing by the use of galvanometric scanners and high precision positioner. As a matter of fact, the use of

\footnotetext{
${ }^{a)}$ Electronic mail: aligokhan.demir@polimi.it

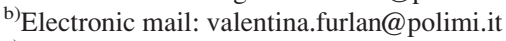

${ }^{\text {c) }}$ Electronic mail: nora.lecis@polimi.it

d) Author to whom correspondence should be addressed; electronic mail: barbara.previtali@polimi.it
}

lasers has been exploited in different ways for biomedical applications, which include laser assisted coatings, laser surface texturing, and laser induced forward transfer (LIFT) on metallic and polymeric materials. ${ }^{6}$

In particular, regarding surface wettability, two states can be identified as hydrophobic and hydrophilic. Wettability is often measured with the contact angle (CA) of the liquid on the surface, which is often water. Contact angles between $0^{\circ}$ and $90^{\circ}$ represent the hydrophilic region and between $90^{\circ}$ and $180^{\circ}$ represent the hydrophobic region. The control of surface wetting is essential for surface adhesion, biocompatibility, and water absorption. Hydrophilic surfaces are often preferred for better adhesion properties and liquid harvesting, whereas hydrophobic surfaces are interesting for self-cleaning surfaces and stiction prevention. Wettability depends directly on surface topography, as increase of surface area and hierarchical organization of surface nanoand microfeatures can either increase or decrease the contact angle. The theory regarding surface wettability has been extensively described in the literature. Some of the key models regarding the link between surface topography and contact angle are summarized in the following. For an ideally flat surface, it is possible to define the apparent contact angle $\left(\theta_{\mathrm{y}}\right)$ by Young's equation ${ }^{7}$

$$
\cos \theta_{\mathrm{y}}=\frac{\gamma_{\mathrm{SG}}-\gamma_{\mathrm{SL}}}{\gamma_{\mathrm{LG}}},
$$

where $\gamma_{\mathrm{SG}}, \gamma_{\mathrm{SL}}$, and $\gamma_{\mathrm{LG}}$ are, respectively, surface tension at solid/gas, solid/liquid, and liquid/gas interfaces. The applicability of Young's equation cannot be extended easily to the real surfaces that are commonly inhomogeneous and rough. Young's model has been further developed by Wenzel to introduce the effect of roughness of surface. The roughness factor (r) was introduced as the ratio between effective surface area and an ideally flat surface area that respects Young's condition. By Wenzel's equation, it is possible to have a relation between the contact angle of ideally flat surface $\left(\theta_{\mathrm{y}}\right)$ and the contact angle of rough surface $\left(\theta_{\mathrm{w}}\right)^{8,9}$ as 


$$
\cos \theta_{\mathrm{w}}=\mathrm{r} \cos \theta_{\mathrm{y}}
$$

with $r>1$. According to this relationship, if an ideally flat surface is hydrophilic $\left(\theta_{\mathrm{y}}<90^{\circ}\right)$, the increase in surface area (r) results in decrease of the apparent contact angel $\left(\theta_{\mathrm{w}}\right)$. On the contrary, an ideally flat surface is initially hydrophobic $\left(\theta_{\mathrm{y}}>90^{\circ}\right)$; the increase in surface area (r) results in increase of the apparent contact angel $\left(\theta_{\mathrm{w}}\right)$. Wenzel's model indicates that the control over surface wettability can be achieved via the control over the surface area, which is usually directly correlated to the surface roughness. In particular, the contact angle of an already hydrophilic surface can be further decreased by increasing surface area.

Another derivation from Young's theory is the model of Cassie-Baxter that takes heterogeneity of phases at the wetting interphase into account. The model introduces the contribution of the gaseous phase as well as the effective surface area. This allows describing heterogeneous wetting between liquid-solid and liquid-gas phases. Such surface structure suspends the liquid with the help of the gaseous phase entrapped in the microcavities of the surface. ${ }^{10,11}$ Cassie-Baxter relationship can be expressed as the following:

$$
\cos \theta_{\mathrm{c}}=\mathrm{f} \cos \theta_{\mathrm{y}}-(1-\mathrm{f}),
$$

where $f$ and $(1-f)$ are the area fractions of the solid and gas phases on the surface. The direct implication of Cassie-Baxter model is that the gaseous phase can contribute to increase the contact angle if the surface microgeometry allows air entrapment. Moreover, the relationship also implies that even highly hydrophilic surfaces can become highly hydrophobic if the surface topography can be manipulated to entrap gaseous phase. This model is usually used to explain wetting behavior of complicated surface structures and the super hydrophobic behavior of surfaces that incorporate hierarchical micro- and nanostructures.

In order to link the wetting behavior to the adhesion performance, the thermodynamic work of adhesion $\mathrm{W}_{\mathrm{a}}$ expressed by Dupré can be used ${ }^{12}$

$$
\mathrm{W}_{\mathrm{a}}=\gamma_{\mathrm{LG}}(\cos \theta+1),
$$

where $\theta$ is the apparent contact angle of the adherent. For an accurate estimation, the contact angle and the surface energy of the adherent are required, but conventionally water contact angles can be used for comparative purposes.

The theoretical analysis shows that in order to achieve good adhesive bonding between the adherent layer and the substrate, the contact angle plays a key role. On the other hand, the surface chemistry and mechanical interlocking effects are other contributing factors. Laser surface texturing can be used to control surface micro- and nanotopography to reduce contact angle, as well as providing increase of area and anchoring points to increase resistance to adhesion failure. Moreover, the processing environment is a determining fact on the final surface chemistry, depending on whether inert or reactive conditions are applied. Magnesium has a high affinity to oxygen and in its presence, especially at elevated temperatures reacts according to ${ }^{13}$

$$
\mathrm{Mg}+\frac{1}{2} \mathrm{O}_{2} \rightarrow \mathrm{MgO}
$$

Due to the formation of a protective oxide film, $\mathrm{Mg}$ exhibits good oxidation resistance in dry air at normal ambient temperature. The presence of water in the environment degrades the film by the formation of a less stable hydrated oxide.

$$
\mathrm{MgO}+\mathrm{H}_{2} \mathrm{O} \rightarrow \mathrm{Mg}(\mathrm{OH})_{2} .
$$

It is attainable that surface oxidation generated through laser structuring in ambient atmosphere might generate $\mathrm{MgO}$ with high affinity to water. Such affinity is expected to cause water spread over the surface, reducing the apparent contact angle and exhibiting highly hydrophilic surfaces.

The use of laser surface texturing for wettability control has been reported on polymeric ${ }^{14,15}$ and metallic materials. ${ }^{16-18}$ The works reporting microsurface textures result in limited changes in contact angle, whereas the use of nanometric surface textures obtained with ripple patterns using ultrashort pulses results in highly hydrophobic surfaces. ${ }^{16-18}$ This phenomenon is the consequence of the air entrapment as explained in Cassie-Baxter model. Moreover, the dependence of contact angle of the textured surfaces on time has been also reported. It has been shown that the hierarchical surface textures composed of micro- and nanofeatures on metallic surfaces were initially hydrophilic $\left(\mathrm{CA}=20^{\circ}\right)$ and in time became hydrophobic $\left(\mathrm{CA}=150^{\circ}\right) .{ }^{16}$ Such change was attributed to the change of surface chemistry.

Among the numerous scientific works, very little information is available regarding the laser surface treatment of $\mathrm{Mg}$ alloys. Taltavull et al. recently reported diode laser heat treatment of AZ91 Mg alloy, by complete or selective surface melting. ${ }^{19}$ The authors reported the change of material microstructure and increase of hardness at the treated layer. Guan et al. reported their extensive results on laser surface treatment of AZ91 and AZ31 alloys. ${ }^{20-23}$ The authors applied surface melting by a microsecond-pulsed Nd:YAG laser and observed improved wettability with an increase in surface energy from 25.82 to $40.78 \mathrm{~mJ} / \mathrm{m}^{2} .{ }^{20}$ With a similar laser system and the same material, they presented improved corrosion behavior along with the microstructural changes. ${ }^{21}$ The same authors studied the interaction of a femtosecond-pulsed laser beam with the AZ31 Mg alloy, where they observed the iridescent effect caused by micro- and nanoripples generated with the laser. ${ }^{22}$ The authors also studied darkening effect induced by oxidation and roughness change using a large excimer laser beam. ${ }^{23}$

On the other hand, chemical processes have been used more often to modify the wettability of $\mathrm{Mg}$ alloys. Han et al. used a procedure based on chemical etching with $\mathrm{HCl}, \mathrm{Ni}$ deposition, and finally surface modification by stearic acid. ${ }^{24}$ The authors obtained low contact angles after the initial chemical etching step, whereas super hydrophobic behavior was present after the final surface modification step. In order 
to achieve super hydrophobic behavior on pure $\mathrm{Mg}$, Wang et al. used a two stepped chemical etching treatment. ${ }^{25}$ The authors obtained a flowerlike microstructure on the surface, which entrapped air in the surface and became water repellent. On the other hand, Liu et al. incorporated laser texturing with chemical etching to generate super hydrophobic surfaces on AZ91 Mg alloy. ${ }^{26}$ The authors combined the microdimensional dimples obtained by laser texturing with nanoscale spikes to obtain such nonwetting behavior.

It can be observed that the common practice in surface modification of $\mathrm{Mg}$ alloys involve the use of chemical etchants. These processes are more prone to environmental changes and are nondeterministic in nature. The etchants are expected to generate material loss within surface modification process, which can be unacceptable for small biomedical implants such as stents. Moreover, the used chemical substances are harmful to the environment; both their use and disposal are problematic. The use of laser surface structuring allows robust, repeatable processing conditions, which can be adapted to local or large area treatments. Moreover, the process is environmentally friendly and requires simple instrumentation that can be easily adapted to industrial production lines. As a matter of fact, for specific cases such as cardiovascular stents, laser surface structuring can be implemented within the same machining setup used for laser cutting of the implant. ${ }^{27}$

In this work, the use of a pulsed fiber laser source to change surface structure of AZ31 Mg alloy is studied to control wetting behavior with the aim to improve adhesion. A single-step strategy based on surface remelting was employed based to generate five different surface types. These surfaces were extensively studied in terms of micro- and nanotopography, surface chemistry, and surface mechanical integrity. The wettability characterization showed effective control of the water contact angle as a function of the surface roughness.

\section{METHODS}

\section{A. Material}

AZ31 magnesium alloy sheets were used throughout the study. The sheets were cold rolled to $0.4 \mathrm{~mm}$ thickness. The measured chemical composition of the material consisted of 3.08 wt.\% Al, 0.98 wt.\% $\mathrm{Zn}$, and balance of $\mathrm{Mg}$. The average surface roughness was measured as $\mathrm{R}_{\mathrm{a}}=0.255 \pm 0.009 \mu \mathrm{m}$ and average distance between the highest peak and lowest valley as $R_{z}=1.771 \pm 0.040 \mu \mathrm{m}$.

\section{B. Laser surface structuring}

A pulsed active fiber laser was used for laser surface structuring (YLP-1/100/50/50 from IPG Photonics, Oxford, MA, USA). The laser source provided a collimated beam at the output, which was manipulated in $\mathrm{X}$ and $\mathrm{Y}$ coordinates by means of a scanner head (TSH 8310 by Sunny Technology, Beijing, China). The scanner head was equipped with an f-theta lens with $100 \mathrm{~mm}$ focal distance that allowed a flat scanning plane (SL-1064-70-100 from
Wavelength Opto-Electronic, Ronar-Smith, Singapore). The main specifications of the used laser system are summarized in Table I. The laser beam was characterized by high quality (beam propagation ratio $\mathrm{M}^{2}=1.7$ ) and a spatial energy distribution very similar to $\mathrm{TEM}_{00}$ (transverse electromagnetic mode). A quasi-Gaussian distribution can be used for modeling the energy distribution within the laser spot. In the case of nanosecond-pulsed laser-material interactions, several effects can be present within the Gaussian energy distribution. Toward the peak of the energy distribution, energy density reaches levels high enough to remove material by ablation. Toward the tails of the energy profile, flat melting and cleaning effects are induced due to lower fluence levels. $^{28}$ Therefore, laser beam was focused $2 \mathrm{~mm}$ above the surface to generate a larger spot and reduce energy intensity on the material surface. This way material removal was avoided and processing conditions yielded melting of a superficial layer. The calculated beam diameter at the focal point was $39 \mu \mathrm{m}$, whereas the spot size $\left(d_{s}\right)$ on the workpiece was $124 \mu \mathrm{m}$. On the other hand, laser pulse energy was maintained between 0.10 and $0.25 \mathrm{~mJ}$.

Laser surface structuring consists of scanning the laser beam on a two dimensional plane to overlap laser pulses on the material (see Fig. 1). The laser source is operated with a set pulse repetition rate (PRR) and the beam scans a line with a given scan speed (v). The overlapping on the scan direction $(\mathrm{R} \%)$ is determined by these two parameters, along with the laser spot size and can be calculated from the following equation:

$$
\mathrm{R} \%=\left(1-\frac{\mathrm{v}}{\mathrm{PRR} \times \mathrm{d}_{\mathrm{s}}}\right) \times 100 .
$$

On the other hand, the overlapping of the successive scan lines depends on the pitch (p). In order to increase the overlapping between lines, pitch was set as $10 \mu \mathrm{m}$ in all experiments. It is also possible to increase the number of passes (S) on the scanned area. In particular, scan passes can be made on different angles to eliminate directionality on the surface. Within this study, single passes were done at $0^{\circ}$, which was defined as parallel to the rolling traces on the material. In multiple passes, the scan angle followed $0^{\circ}, 90^{\circ}$, $45^{\circ}$, and $135^{\circ}$ (as shown in Fig. 2).

TABLE I. Main specifications of the laser surface structuring system employed in the study.

\begin{tabular}{lc}
\hline \hline Wavelength & $1064 \mathrm{~nm}$ \\
Maximum average power & $50 \mathrm{~W}$ \\
Pulse duration & $250 \mathrm{~ns}$ \\
Pulse repetition rate & $20-80 \mathrm{kHz}$ \\
Maximum pulse energy & $1 \mathrm{~mJ}$ \\
Maximum peak power & $9 \mathrm{~kW}$ \\
$\mathrm{M}^{2}$ & 1.7 \\
Collimated beam diameter & $5.9 \mathrm{~mm}$ \\
Focal lens & $100 \mathrm{~mm}$ \\
Focused beam diameter & $39 \mu \mathrm{m}$ \\
\hline \hline
\end{tabular}




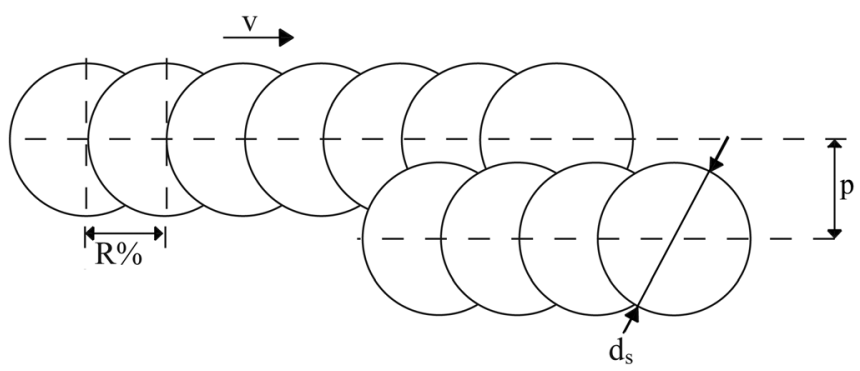

FIG. 1. Overlapping in the scan direction and between scan lines. Single circles represent laser irradiated area by each pulse. Pulses are overlapped by $\mathrm{R} \%$ in scan direction and by p between consecutive scan lines.

The energetic parameters involved in laser surface structuring can be unified under a single parameter such as average line fluence $\left(\mathrm{F}_{1}\right)$. This parameter represent the intensity of average laser energy released on scan line and is expressed by the following equation:

$$
\mathrm{F}_{1}=\frac{\mathrm{E} \times \mathrm{PRR}}{\mathrm{v} \times \mathrm{d}_{\mathrm{s}}} .
$$

As can be observed from Eq. (8), average line fluence does not take the number of passes into account and averages the effect of time dependant characteristics of thermal surface structuring process. In this study, average line irradiance was used for comparing the energetic conditions to obtain distinct surface structures.

After initial experiments, which are not reported here for the sake of brevity, five parameter sets were determined that generated distinct surface topographies. The surfaces were obtained with scan speeds of 25 and $100 \mathrm{~mm} / \mathrm{s}$, and pulse repetition rates of 20 and $70 \mathrm{kHz}$. These parameters allowed very high overlapping on the scan direction between $96 \%$ and $99 \%$. With the employed spot size and pulse energy levels, average line irradiances varied between 16 and $160 \mathrm{~J} / \mathrm{cm}^{2}$. All laser surface structuring experiments were carried out in ambient conditions, without the use of a process gas. Prior to laser processing, the samples were cleaned in ultrasonic bath in acetone for $10 \mathrm{~min}$ and dried in air.
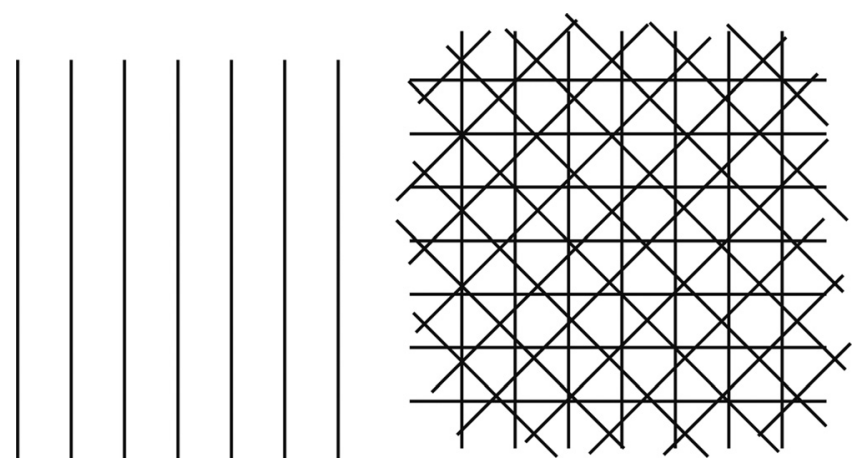

FIG. 2. Scheme of the employed scan angle strategies with single pass and four passes. The single pass consists of scan lines distanced by pitch (p) and aligned parallel to the rolling direction of the AZ31 sheet, corresponding to $0^{\circ}$. When four consecutive passes are applied, scans were applied at $0^{\circ}, 90^{\circ}$, $45^{\circ}$, and $135^{\circ}$ consecutively, with the same pitch value.
TABLE II. Laser surface structuring parameters used to generate the five distinct surface morphologies.

\begin{tabular}{lccccc}
\hline \hline Surface type & Polished & Transition & Worms & Buds & Cauliflower \\
\hline Pulse energy, E (mJ) & 0.10 & 0.10 & 0.25 & 0.25 & 0.25 \\
Pulse repetition rate, & 20 & 20 & 20 & 20 & 70 \\
PRR (kHz) & & & & & \\
Scan speed, v (mm/s) & 100 & 100 & 100 & 25 & 100 \\
$\begin{array}{l}\text { Number of passes, S } \\
\text { Pitch, p }(\mu \mathrm{m})\end{array}$ & 1 & 4 & 1 & 4 & 4 \\
Focal position, f $(\mathrm{mm})$ & 2 & 10 & 10 & 10 & 10 \\
$\begin{array}{l}\text { Laser spot diameter, } \\
\mathrm{d}_{\mathrm{s}}(\mu \mathrm{m})\end{array}$ & 124 & 124 & 124 & 124 & 2 \\
$\begin{array}{l}\text { Overlap on scan } \\
\text { direction, R\% }\end{array}$ & 96.0 & 96.0 & 96.0 & 99.0 & 98.9 \\
$\begin{array}{l}\text { Average line fluence, } \\
\mathrm{F}_{1}\left(\mathrm{~J} / \mathrm{cm}^{2}\right)\end{array}$ & 16.1 & 16.1 & 40.2 & 160.9 & 140.8 \\
Process gas & None & None & None & None & None \\
\hline \hline
\end{tabular}

Table II reports the used laser structuring parameters along with the coded names of the obtained surface structures.

\section{Morphology analysis}

Surface morphology was characterized via SEM (EVO-50, from Carl Zeiss, Oberkochen, Germany). Imaging was carried out in both secondary electron and backscattered electron (BSE) modes. Surface roughness was measured with an optical 3D profilometer based on focus variation microscopy (InfiniteFocus from Alicona Imaging GmbH, Graz, Austria). The acquisition area was limited to $1.3 \mathrm{~mm} \times 0.2 \mathrm{~mm}$. Five lines along the long axis were taken for measuring average surface roughness $\left(\mathrm{R}_{\mathrm{a}}\right)$ and average distance between the highest peak and lowest valley $\left(\mathrm{R}_{\mathrm{z}}\right)$.

\section{Scratch test}

Scratch tests were done to characterize the adherence of the structured layer to the bulk of the material. The tests were performed using a scratch tester (Microcombi Platform from CSM Instruments, Peseux Switzerland) with an increasing load from 0.3 to $30 \mathrm{~N}$. Scratches were made 3 $\mathrm{mm}$ in length using a diamond Rockwell indenter with a spherical tip radius of $200 \mu \mathrm{m}$ sliding at a constant speed of $1.26 \mathrm{~mm} / \mathrm{min}$. Scratch tracks were aligned parallel to the first scan angle (i.e., scratch tracks were aligned to $0^{\circ}$ ). A critical load $\left(\mathrm{L}_{\mathrm{c}}\right)$ was identified corresponding to the load for the detachment of the structured surface from the material bulk. $\mathrm{L}_{\mathrm{c}}$ was determined from optical microscopy images taken with the same machinery that could correlate the image position to the applied load. Scratch test was applied also to nonstructured AZ31 surface, where the $\mathrm{L}_{\mathrm{c}}$ was determined as the load required for detaching natural surface oxide.

\section{E. Chemical composition}

X-ray diffractometry was employed to characterize the chemical composition of the structured surfaces. (X'Pert Pro from PANalytical, Almelo, the Netherlands) with $\mathrm{Cu}$ 
cathode source and a silicon strip detector (X'celerator from PANalytical, Almelo, the Netherlands). Measurement interval was $5^{\circ}-120^{\circ}$, with an incremental step of $0.016^{\circ}$.

\section{F. Water contact angle}

Wetting behavior of the substrates has been analyzed to define an adhesion performance indicator of the five selected surfaces. A ring/plate tensiometer (Te 3 from Lauda, LaudaKönigshofen, Germany) was used to measure the contact angle $(\theta)$ of the nonstructured and laser structured surfaces based on Wilhelmy Plate Method. Test liquid was distilled water with a known surface tension $(\gamma)$ of $72 \mathrm{mN} / \mathrm{m}$. For the tests, square samples with $10 \mathrm{~mm} \times 10 \mathrm{~mm}$ dimensions, laser structured on both faces were prepared. The samples were immersed in the test liquid with $0.01 \mathrm{~mm} / \mathrm{s}$ constant speed. During the preliminary measurements, no significant change in the water contact angle as a function of the immersion angle was observed. Therefore, the samples were immerged at $0^{\circ}$, i.e., the rolling direction was parallel to the water surface. The forces retracting the samples were measured with the load cell of the machine for $150 \mathrm{~s}$ with $1 \mathrm{~Hz}$ acquisition rate after the first contact with the test liquid. The values were then converted to the contact angle using the simplified Wilhelmy equation ${ }^{29}$

$$
\cos (\theta)=\frac{\mathrm{F}}{1 \times \gamma},
$$

where 1 is the wetted perimeter, which was $20.8 \mathrm{~mm}$ for all the samples. The average of the final three measurement points was taken as the static contact angle of the measurement. Three replicates were prepared for nonstructured and structured samples.

\section{RESULTS AND DISCUSSION}

Figure 3 reports the surface morphologies obtained with the SEM. It can be observed that there is a notable increase in the surface roughness and surface area moving toward the more energetic laser surface structuring conditions. In particular, the lowest energetic conditions $(E=0.10 \mathrm{~mJ}$, PRR $=20 \mathrm{kHz}, \mathrm{v}=100 \mathrm{~mm} / \mathrm{s}, \mathrm{F}_{1}=16.1 \mathrm{~J} / \mathrm{cm}^{2}, \mathrm{~S}=1$ ) resulted in a smoother surface structure eliminating some of the surface defects [see Figs. 3(a) and 3(b)]. Hence, this surface structure was named "polished." Increasing the number of passes to 4 with the same energetic conditions $(S=4)$, the surface becomes less homogeneous. It is observed that along the rolling direction, clusters of sub micrometer features nucleate [see Figs. 3(c) and 3(d)]. This orientation of cluster nucleation might be induced due to the local internal stress fields inherited from the rolling process. The thermal effect of the laser beam can induce different stress fields along the rolling direction, causing this type of local morphology change. With increased pulse energy $(\mathrm{E}=0.25 \mathrm{~mJ})$ and a single pass $\left(F_{1}=40.2 \mathrm{~J} / \mathrm{cm}^{2}, S=1\right)$, the surface of AZ31 becomes rougher, although the surface texture appears more homogeneous. At these energetic conditions surface melting
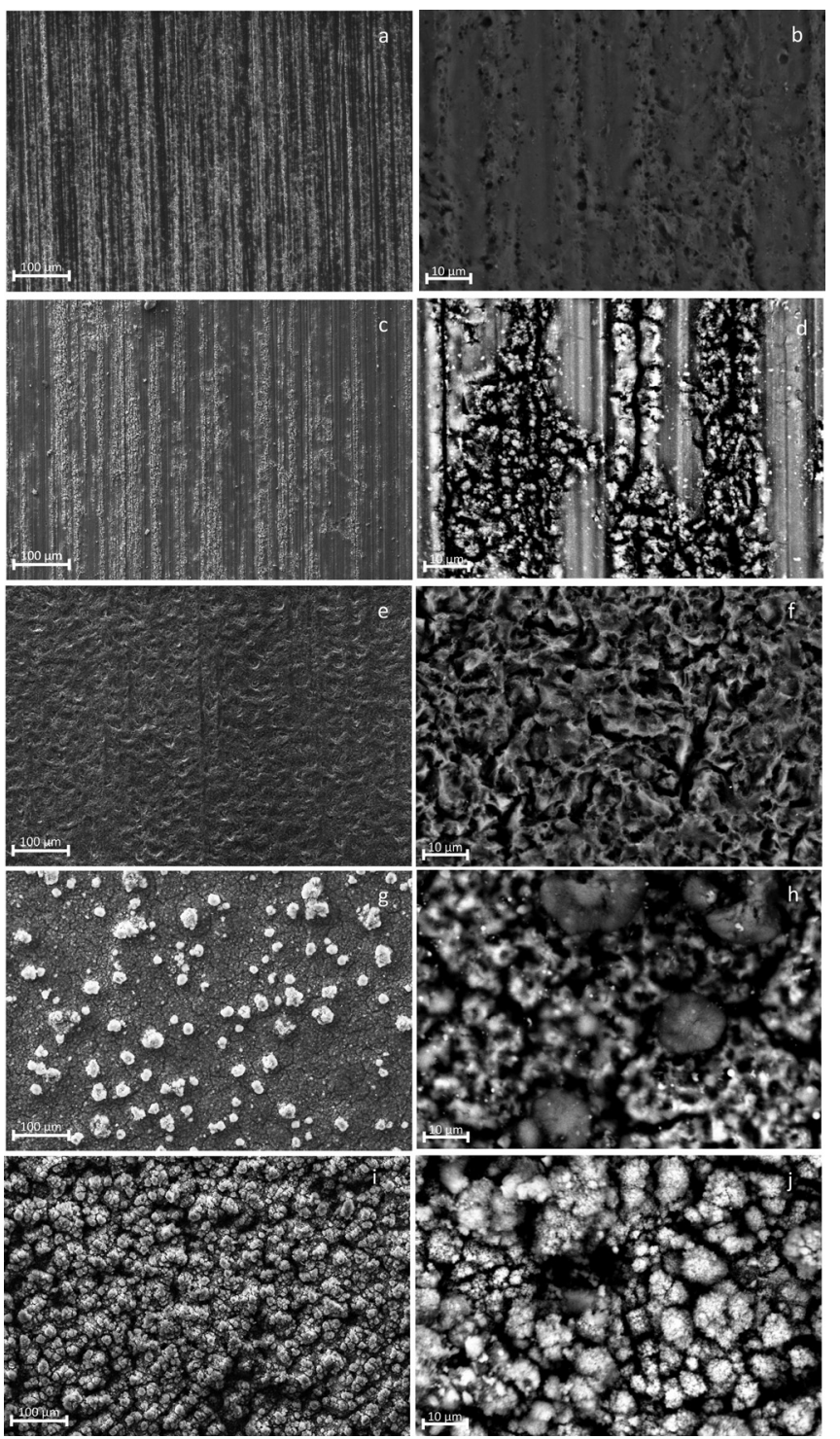

FIG. 3. SEM images of the laser structured AZ31 surfaces. Images were obtained with secondary electrons in lower magnification and back scattered electrons in high magnification. The structures were coded as polished [(a) and (b)], transition [(c) and (d)], worms [(e) and (f)], buds [(g) and (h)], and cauliflower [(i) and (j)] according to the observed surface morphology and its resemblance.

is expected to occur completely in a thicker section [see Fig. $3(\mathrm{e})]$. By rapid heating and cooling cycles, the material resolidifies to a wormlike structure [as seen in Fig. 3(f)]; hence, it is nominated as "worms." Reduced scan speed ( $\mathrm{v}=25$ $\mathrm{mm} / \mathrm{s}$ ) with the previous energy level and four consecutive passes $\left(F_{1}=160.9 \mathrm{~J} / \mathrm{cm}^{2}, \quad \mathrm{~S}=4\right)$ generate a surface that exhibits spherical clusters of material above the rough surface [see Fig. $3(\mathrm{~g})]$. The clusters are expected to be $\mathrm{MgO}$, which is confirmed by the darker shades of gray seen in BSE images [see Fig. 3(h)]. The clusters that resemble cotton buds exhibit nanometer-sized features. The surface type is therefore called "buds." The final laser surface structuring conditions used higher scan speed $(\mathrm{v}=100 \mathrm{~mm} / \mathrm{s})$ with higher pulse repetition rate $(\mathrm{PRR}=70 \mathrm{kHz})$, which in return 
generated lower average line fluence compared to the previous case $\left(\mathrm{F}_{1}=140.8 \mathrm{~J} / \mathrm{cm}^{2}, \mathrm{~S}=4\right)$. The surface obtained with these parameters was characterized by the complete coverage of clustered structures. The complete surface transformation is expected to be due to a faster thermal cycle induced by increased pulse repetition rate, despite lower average line fluence. The structures appeared in fractal form accompanying both micrometer and nanometer features. The surface type was named "cauliflower" due to the fractal resemblance.

Although the thermodynamic cycles induced by the laser treatment are expected to yield different effects compared to conventional heat sources, it is useful to consider some key physical properties of this alloy to better hypothesize the mechanisms behind the structure formation. AZ31 melts at $905 \mathrm{~K}$ and boils at $1363 \mathrm{~K} .{ }^{30}$ Moreover above $873 \mathrm{~K}$, oxidation of $\mathrm{Mg}$ becomes extremely fast, leading to ignition. ${ }^{13} \mathrm{On}$ the other hand, ablation plume was visible during the machining, which is expected to contain ionized plasma of $\mathrm{Mg}$ and other species ionized from the ambient air. The plasma temperature in laser welding of $\mathrm{Mg}$ has been estimated to be as high as $8000 \mathrm{~K}^{31}$ Although the machining conditions are much different in the employed surface structuring strategies, the plasma temperature can be expected to be an order of magnitude higher than the boiling temperature of AZ31. Intriguingly, the laser surface structuring applied in this study never resulted in ignition, and material loss due to vaporization was not observed. As a matter of fact, the plasma plume can be expected to increase the pressure locally around the processing zone, preventing ignition to occur although higher temperatures are reached. Regarding the oxidation kinetics, below $723 \mathrm{~K}$ in dry oxygen, a protective oxide film on magnesium is generated. ${ }^{13}$ On the other hand at $723 \mathrm{~K}$, the formation of a porous oxide layer is reported in commercial $\mathrm{Mg}$ alloys. Initially, a thin layer of $\mathrm{MgO}$ is generated which grows in parabolic kinetics and acts as diffusion barrier. After the reach of a certain thickness, tensile stresses are generated, which cause the $\mathrm{MgO}$ film to crack and permit the access of oxygen to the metal surface. In this second phase, the thickness of the $\mathrm{MgO}$ layer grows much faster with a porous structure. The time scale reported for such mechanism is in the order of hours for AM50 ingots. However, given the higher temperature gradients, localized energy input, and higher pressure caused by the ablation plume of the laser processing conditions in this work, the creation of porous structures, especially in the significantly oxidized buds and cauliflower surface types, can be linked to such mechanism.

3D surface profiles of the obtained surfaces are reported in Fig. 4. It can be observed that the surface roughness increases as the surface features evolve from flat topography of the polished to the clustered topography of the cauliflower types. The images were used to measure to the surface roughness parameters, which confirm the observations. Specifically for the case of polished surface type, both $\mathrm{R}_{\mathrm{a}}$ and $\mathrm{R}_{\mathrm{z}}$ parameters are lower than the initial values $\left(R_{a}=0.219 \pm 0.009 \mu \mathrm{m}\right.$, $\left.R_{z}=1.303 \pm 0.047 \mu \mathrm{m}\right)$. The reduction in the $R_{z}$ parameter confirms that the gentle surface melting allowed the elimination of the crests on the surface. The surface roughness parameters are higher than the nonstructured surfaces in the range between transition and cauliflower surface types. In this range, surface roughness increases from $\mathrm{R}_{\mathrm{a}}=0.4 \mu \mathrm{m}, \mathrm{R}_{\mathrm{z}}=2.8 \mu \mathrm{m}$ to $\mathrm{R}_{\mathrm{a}}=4.3 \mu \mathrm{m}, \mathrm{R}_{\mathrm{z}}=23.7 \mu \mathrm{m}$. For the complete list of surface roughness measurements, refer Table III. It is also noticed that the variation in measured surface roughness parameter increases notably moving toward rougher surfaces. Although the used processing method was deterministic, the obtained surface structures were stochastic. As the surface structure becomes more complicated, surface area increases in also a stochastic manner, which reflects to higher variation of the measured surface parameters.

Figure 5 shows the optical images of the scratch tracks belonging to the different surfaces. The images represent the instance of the critical load $\left(\mathrm{L}_{\mathrm{c}}\right)$. The critical load of the nonstructured surface was $14.0 \pm 1.4 \mathrm{~N}$, which has been considered to be the reference condition. Polished surface type behaved very similar to the nonstructured surface $\left(\mathrm{L}_{\mathrm{c}}=14.0 \pm 1.4 \mathrm{~N}\right)$ under the scratch test. The gentle surface melting conditions, therefore, did not appear to alter the surface integrity. On the other hand, the transition type showed a notable decrease in the critical load $\left(\mathrm{L}_{\mathrm{c}}=8.0 \pm 0 \mathrm{~N}\right)$. The sub micrometer features along the rolling direction are expected to lower the scratch resistance. In the case of worms surface type, scratch resistance was not significantly altered $\left(\mathrm{L}_{\mathrm{c}}=13.5 \pm 0.7 \mathrm{~N}\right)$. This confirms that the surface texture was homogeneously distributed and surface integrity was maintained. The buds surface type showed a slight decrease in the critical load $\left(\mathrm{L}_{\mathrm{c}}=11.5 \pm 0.7 \mathrm{~N}\right)$, and within the scratch track, small cracks were observable. The oxidation during the laser structuring in ambient atmosphere is expected to render the surface more fragile. Similar behavior was present in the case of cauliflower surface type, also critical load was much similar to the one belonging to buds $\left(\mathrm{L}_{\mathrm{c}}=11.0 \pm 1.4 \mathrm{~N}\right)$. Overall, the surface integrity was confirmed for polished and worms surface types, it was acceptable for buds and cauliflower, whereas transition surface were found to be damaged by the laser treatment.

In Fig. 6, the results of the XRD analysis are shown. Several peaks belonging to $\mathrm{Mg}$ and $\mathrm{MgO}$ were identified between $30^{\circ}$ and $75^{\circ}$, confirming surface oxidation occurring at laser processing in ambient conditions. A strong crystal texture at [002] plane was found belonging to the material bulk at $34.5^{\circ}$. The other $\mathrm{Mg}$ peaks around $32.5^{\circ}, 37^{\circ}$, and $70.5^{\circ}$ decreased with increased roughness of the surface type, whereas the $\mathrm{MgO}$ peaks around $41.5^{\circ}$ and $61.5^{\circ}$ increased. The amount of surface oxide was similar in polished and transition surface types and less than the others. Buds and cauliflower types showed almost identical oxidation level and their oxide component was found to be the highest. The oxidation level of worms type was found to be in the middle of the two groups.

The behavior of water contact angle of the different surfaces during the Wilhelmy plate tests are depicted in Fig. 7. It can be observed that all the surfaces were in hydrophilic 

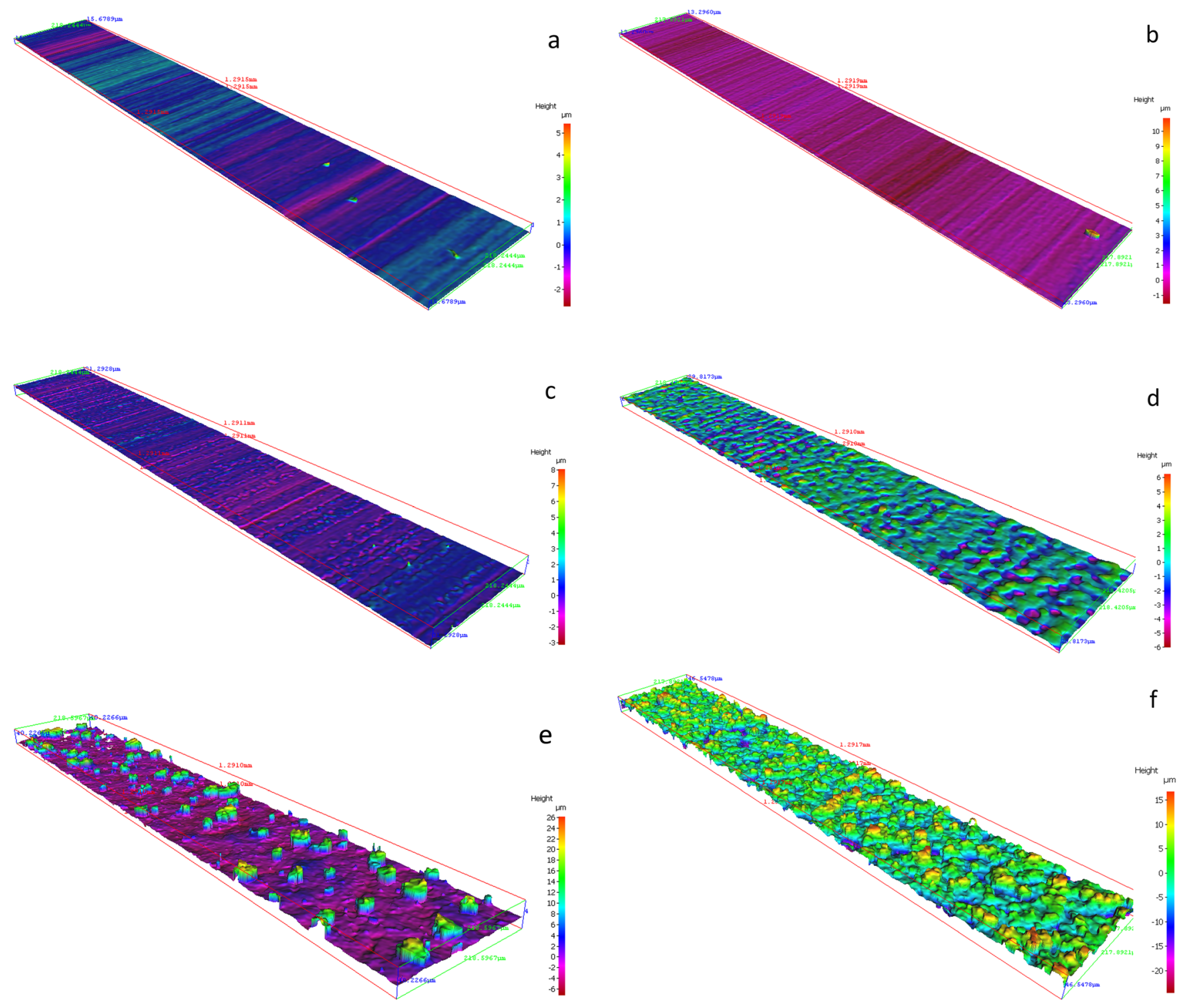

FIG. 4. (Color online) 3D surface profiles of the nonstructured and laser structured AZ31 surfaces. Acquired image area was $1.3 \mathrm{~mm} \times 0.2 \mathrm{~mm}$. Color scales represent height. (a) Nonstructured, (b) polished, (c) transition, (d) worms, (e) buds, (f) cauliflower.

range $\left(\theta<90^{\circ}\right)$. The surfaces with higher contact angles, nonstructured, polished, and transition types showed an asymptotic decay and stabilized around their final values before the end of the test. This is attributed to the fact that the first contact of the sample was on the sample edge, which does not necessarily possess the same surface morphology with the surface. The surface type with the lowest contact angle was worms, which showed a sudden drop from higher contact angle values to around $20^{\circ}$ and stabilized quickly. Such behavior was not present in the case of buds and cauliflower surface types.

In Fig. 8, the static water contact angles of the surfaces were plotted against their corresponding average surface roughness values as seen. Two distinct zones are observed along the roughness axis. The first zone was up to $\mathrm{R}_{\mathrm{a}}=1.1 \mu \mathrm{m}$, where the water contact angle decreased with

TABLE III. Summary of surface characterization results. The reported values are average \pm standard deviation.

\begin{tabular}{|c|c|c|c|c|c|c|}
\hline Surface type & Nonstructured & Polished & Transition & Worms & Buds & Cauliflower \\
\hline $\mathrm{R}_{\mathrm{a}}(\mu \mathrm{m})$ & $0.255 \pm 0.009$ & $0.219 \pm 0.009$ & $0.362 \pm 0.023$ & $1.069 \pm 0.045$ & $3.021 \pm 0.764$ & $4.335 \pm 0.471$ \\
\hline $\mathrm{R}_{\mathrm{z}}(\mu \mathrm{m})$ & $1.771 \pm 0.040$ & $1.303 \pm 0.047$ & $2.745 \pm 0.423$ & $6.092 \pm 0.526$ & $18.221 \pm 2.379$ & $23.729 \pm 2.329$ \\
\hline $\mathrm{L}_{\mathrm{C}}(\mathrm{N})$ & $14.0 \pm 1.4$ & $14.0 \pm 1.4$ & $8.0 \pm 0$ & $13.5 \pm 0.7$ & $11.0 \pm 1.4$ & $11.5 \pm 0.7$ \\
\hline$\theta$ & $61.3^{\circ} \pm 3.5^{\circ}$ & $68.8^{\circ} \pm 6.3^{\circ}$ & $59.3^{\circ} \pm 3.0^{\circ}$ & $21.6^{\circ} \pm 3.0^{\circ}$ & $30.0^{\circ} \pm 4.3^{\circ}$ & $35.5^{\circ} \pm 1.9^{\circ}$ \\
\hline
\end{tabular}



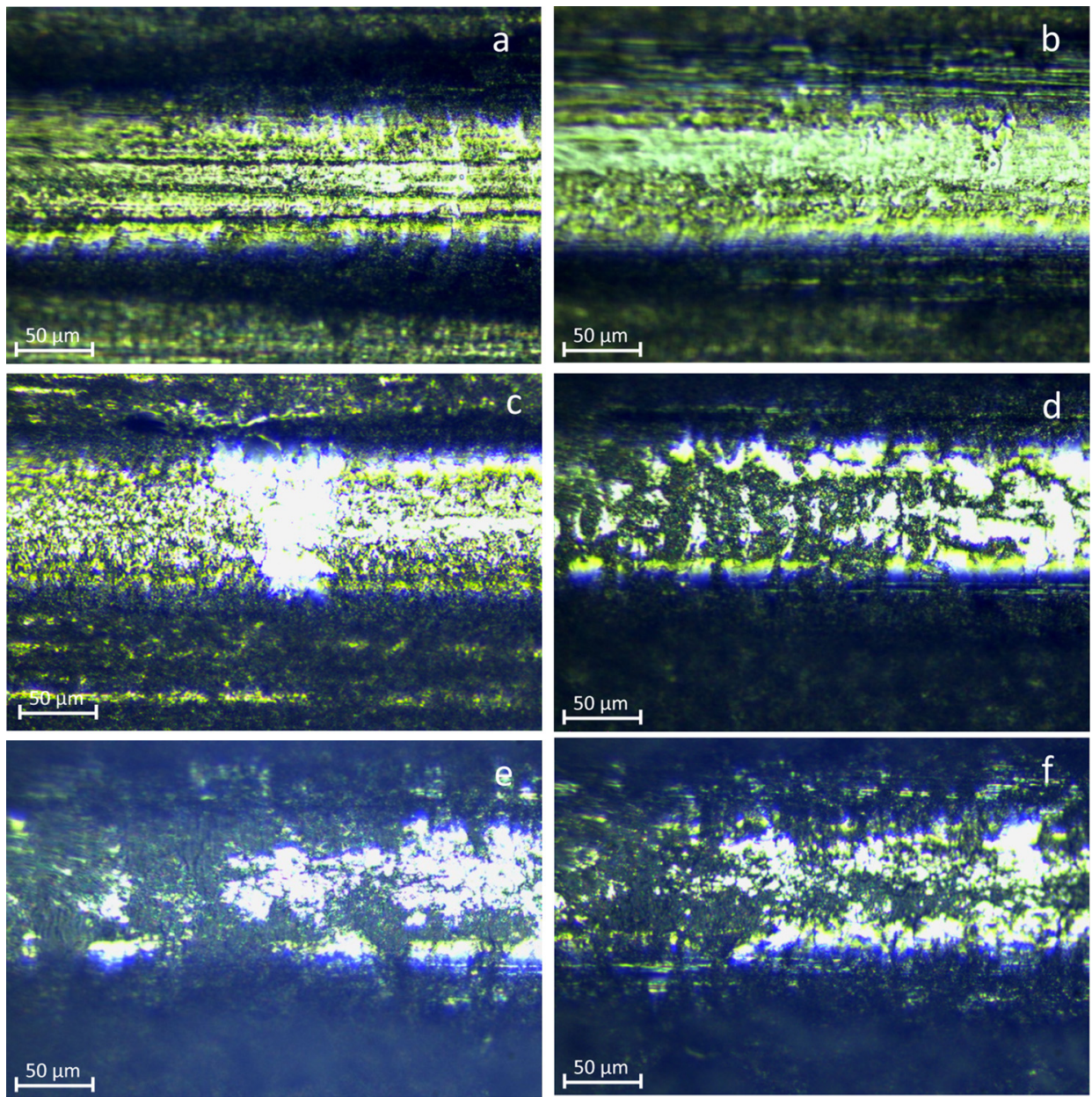

Fig. 5. (Color online) Scratch tracks of the nonstructured and laser structured AZ31 surfaces. The images were obtained at the instance of critical load for surface detachment $\left(\mathrm{L}_{\mathrm{c}}\right.$ ). (a) Nonstructured at $15 \mathrm{~N}$, (b) polished at $15 \mathrm{~N}$, (c) transition at $8 \mathrm{~N}$, (d) worms at $14 \mathrm{~N}$, (e) buds at $10 \mathrm{~N}$, (f) cauliflower at $10 \mathrm{~N}$.

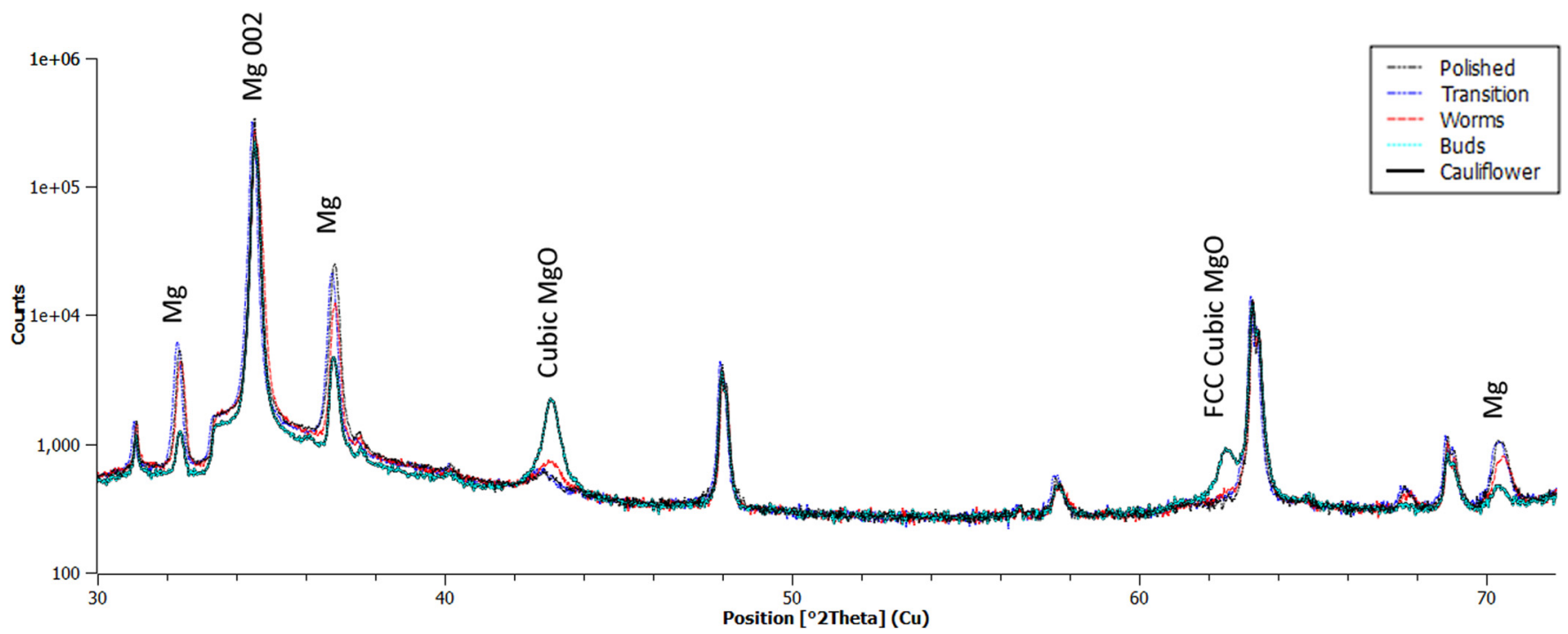

FIG. 6. (Color online) XRD patterns of the laser structured AZ31 surfaces. The most significant $\mathrm{Mg}$ and $\mathrm{MgO}$ peaks are identified. The patterns indicate increasing amount of $\mathrm{MgO}$ with the increase of laser energetic conditions. 


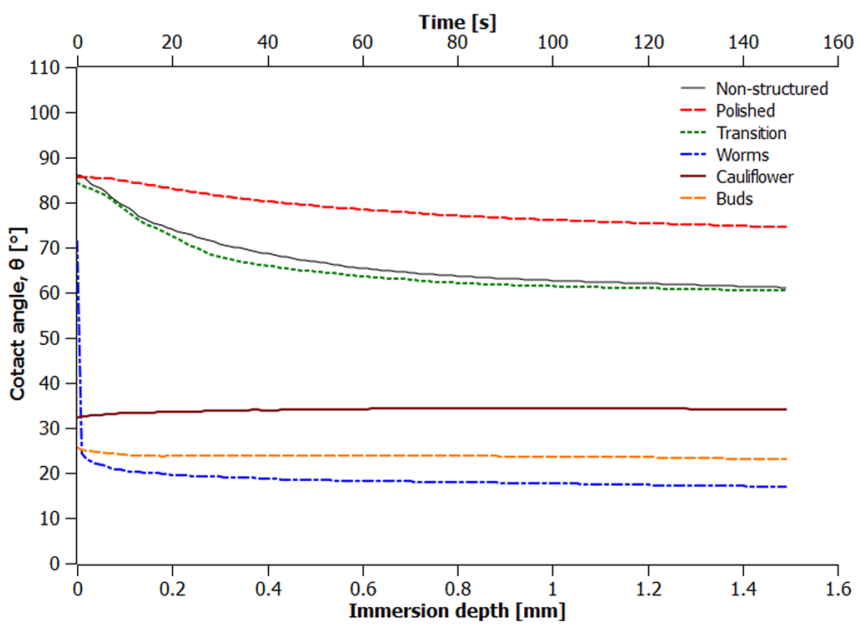

FIG. 7. (Color online) Contact angles of the nonstructured and laser structured AZ31 surfaces as a function of immersion depth measured with Wilhelmy plate method. The specimens were immersed up to $1.5 \mathrm{~mm}$ depth and static contact angle was defined as the average of the final three values.

the increase of the surface roughness. On the other hand, in the range where $\mathrm{R}_{\mathrm{a}}>1.1 \mu \mathrm{m}$, an increase in the contact angle is visible. Two distinct phenomena are expected to contribute in parallel to the change in the contact angle. The first contribution belongs to the increase in the real surface area as a function of the surface roughness, which is coherent with Wenzel's theory. Surface oxidation is not expected to contribute within this region, since XRD analyses showed similar low amount of $\mathrm{MgO}$. Moreover, the increase in the contact angle of the polished surface indicated that the role of surface area is the dominant factor over the change of chemistry. As a matter of fact if the surface chemistry was

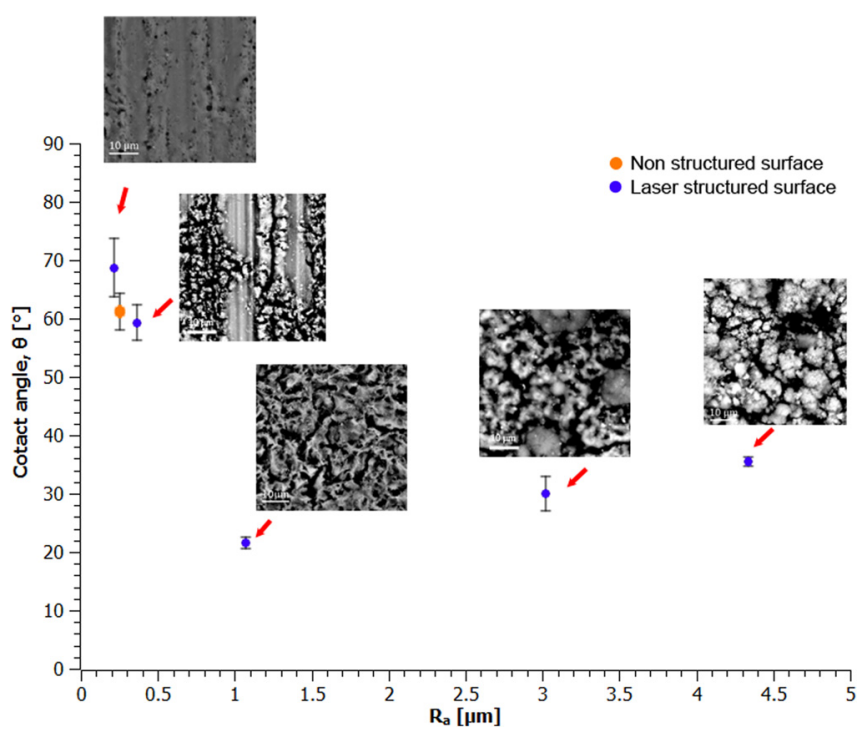

FIG. 8. (Color online) Contact angles of the nonstructured and laser structured AZ31 surfaces as a function average surface roughness. The increase of surface roughness up to $\mathrm{R}_{\mathrm{a}}=1.1 \mu \mathrm{m}$ decreases water contact angle (Wenzel behavior). In the following range of surface roughness where $\mathrm{R}_{\mathrm{a}}>1.1 \mu \mathrm{m}$, water contact angle depends both on chemical and geometrical factors. predominant in this region, polished surface, which is more oxidized compared to the nonstructured surface, should have been more hydrophilic. On the other hand, the surface roughness range where $R_{a}>1.1 \mu \mathrm{m}$ corresponds to the region where both surface chemistry and geometry change significantly. The hierarchical surface structures incorporate both micro- and nanofeatures, which usually result in a super hydrophobic behavior due to the entrapment of air as expressed in Cassie-Baxter relationship. However, this region also corresponds to significant surface oxidation as indicated in the XRD analyses. It is hypothesized that in this region, the dominant factor over the apparent contact angle is the change of chemistry. The chemical reaction between the oxidized surface $(\mathrm{MgO})$ and water causes spread of the liquid phase. The heterogeneous wetting may contribute to increase the contact angle at the same time; however, the sum of the contribution results in hydrophilic behavior. If the chemical affinity of $\mathrm{MgO}$ can be assumed to generate complete water spread over an ideally flat surface $\left(\theta_{\mathrm{y}}=0\right)$, it is possible to calculate the air fraction at the liquid-solid interface ( $1-\mathrm{f}$ ) by rewriting Eq. (3) and using the apparent contact angles of these surfaces $\left(\theta_{\mathrm{c}}\right)$. Accordingly, the entrapped air fraction for buds surface type would be $6.7 \%$ $\left(\theta_{\mathrm{c}}=30.0^{\circ}\right)$, and $9.3 \%\left(\theta_{\mathrm{c}}=35.5^{\circ}\right)$ for cauliflower surface type. The results of all surface roughness, scratch test, contact angle measurements are summarized in Table III.

\section{CONCLUSIONS}

This paper presents the use of a pulsed fiber laser for the surface structuring of AZ31 Mg alloy. Laser process parameters were set to melt a superficial layer rather than removing material to change the surface morphology. The energetic conditions generated different thermal cycles on the material, which smoothened the surface with low average line fluence and increased the surface roughness with increased line fluence compared to the nonstructured surface. The laser surface structuring generated nanometric features as well as change of micrometric surface roughness. The surfaces were evaluated in terms of their adherence to the material bulk via scratch tests and in terms of surface oxidation by XRD. The results showed that the intermediate surface morphology named transition was damaged by the laser treatment, since it showed lower $\mathrm{L}_{\mathrm{c}}$ compared to the nonstructured AZ31. On the other hand, highly rough buds and cauliflower surface types showed higher amount of surface oxidation due to laser processing in ambient air. Among the remaining surface types, polished showed increased water contact angle with reduced surface area, whereas worms surface type showed the lowest water contact angle with increased surface area. The region between the polished and worms surface types $\left(R_{a}=0.2-1 \mu \mathrm{m}\right)$ fit well the Wenzel equation. Between worms and cauliflower surface types $\left(R_{a}>1.1 \mu \mathrm{m}\right)$, the contact angle increased. At this point, both geometrical and chemical aspects were considered to contribute to the change in the apparent contact angle. The oxidized surface increased the affinity to reduce the contact angle, whereas 
the complex surface structure was expected to include entrapped air in the surface and, therefore, increase the contact angle. Within this range, the surfaces remained hydrophobic implying that the contribution of the surface chemistry was the dominant factor over the surface geometry. From this perspective, worms surface type appears to be the best candidate to adhere a polymeric layer on the stent surface, since it reduced contact angle without generating surface damage and excessive oxidation.

The results confirm the effectiveness of a single step laser surface structuring with a nanosecond-pulsed fiber laser on the control of surface wettability. Although the surfaces obtained within this study were of stochastic nature, the applied processing strategies were deterministic and all are based on digital computer adided design and manufacturing (CAD/CAM) systems. This factor opens up many possibilities for flexible surface structuring with simple machinery in industrial scale. Laser surface structuring can be applied on complex free forms on the whole surface or in patches where it is required. Although the main objective of this study has been to identify the potentiality of the use of laser surface structuring to improve surface adhesion by controlling surface wettability of AZ31 Mg alloy, laser surface structuring of $\mathrm{Mg}$ alloys can be extended to other implants such as orthopedic ones. The control on the wetting behavior can also be exploited to control the cell integration and adherence of the implant material to the body tissue. Although the present results are highly promising for future applications, more studies regarding the corrosion rate, cell, and tissue response are required before reaching implantation state. Future works will be also devoted to detach chemical and morphological changes caused by the laser structuring by processing in inert gas atmosphere. More comprehensive studies on the mechanical behavior of the structured surfaces are also planned.

\section{ACKNOWLEDGMENTS}

The authors acknowledge the support from Québec Government for partially funding the research under Project Number 08.205 "Métaux nano-structurés dégradables pour applications pédiatriques" within "Québec-Italie 2013-2015" framework. The authors also wish to thank Luca Di Landro for the availability to use the tensiometer and Paola Bassani for her help with the XRD analysis. The authors declare that they have no competing interests.

${ }^{1}$ M. P. Staiger, A. M. Pietak, J. Huadmai, and G. Dias, Biomaterials 27, 1728 (2006).

${ }^{2}$ F. Witte, Acta Biomater. 6, 1680 (2010).

${ }^{3}$ P. Zartner, R. Cesnjevar, H. Singer, and M. Weyand, Catheter. Cardiovasc. Interv. 66, 590 (2005).

${ }^{4}$ H. Hermawan, D. Dubé, and D. Mantovani, Acta Biomater. 6, 1693 (2010).

${ }^{5}$ J. Yang, F. Cui, and I. S. Lee, Ann. Biomed. Eng. 39, 1857 (2011).

${ }^{6}$ A. Kurella and N. B. Dahotre, J. Biomater. Appl. 20, 5 (2005).

${ }^{7}$ G. Whyman, E. Bormashenko, and T. Stein, Chem. Phys. Lett. 450, 355 (2008).

${ }^{8}$ R. N. Wenzel, Ind. Eng. Chem. 28, 988 (1936).

${ }^{9}$ B. H. Luo, P. W. Shum, Z. W. Zhou, and K. Y. Li, Surf. Coat. Technol. 205, 2597 (2010).

${ }^{10}$ A. B. D. Cassie and S. Baxter, Trans. Faraday Soc. 40, 546 (1944).

${ }^{11}$ A. J. B. Milne and A. Amirfazli, Adv. Colloid Interface Sci. 170, 48 (2012).

${ }^{12}$ A. Baldan, J. Mater. Sci. 39, 1 (2004).

${ }^{13}$ D. Elizer and H. Alves, in Handbook of Materials Selection, edited by M. Kutz (John Wiley \& Sons, Inc., New York, 2002), pp. 267-291.

${ }^{14}$ D. G. Waugh, J. Lawrence, and E. M. Brown, Opt. Lasers Eng. 50, 236 (2012).

${ }^{15}$ D. G. Waugh and J. Lawrence, Appl. Surf. Sci. 257, 8798 (2011).

${ }^{16}$ A.-M. Kietzig, S. G. Hatzikiriakos, and P. Englezos, Langmuir 25, 4821 (2009).

${ }^{17}$ M. Silvennoinen, J. Laser Micro/Nanoeng. 5, 97 (2010).

${ }^{18} \mathrm{P}$. Bizi-Bandoki, S. Benayoun, S. Valette, B. Beaugiraud, and E. Audouard, Appl. Surf. Sci. 257, 5213 (2011).

${ }^{19}$ C. Taltavull, B. Torres, A. J. López, P. Rodrigo, and J. Rams, Surf. Coat. Technol. 222, 118 (2013).

${ }^{20}$ Y. C. Guan, W. Zhou, and H. Y. Zheng, Surf. Rev. Lett. 16, 801 (2009).

${ }^{21}$ Y. C. Guan, W. Zhou, Z. L. Li, and H. Y. Zheng, Mater. Des. 52, 452 (2013).

${ }^{22}$ Y. C. Guan, W. Zhou, Z. L. Li, and H. Y. Zheng, J. Phys. D: Appl. Phys. 46, 425305 (2013).

${ }^{23}$ Y. C. Guan, W. Zhou, Z. L. Li, and H. Y. Zheng, Opt. Lasers Eng. 52, 35 (2014).

${ }^{24}$ M.-H. Han, Y.-H. Park, J.-W. Hyun, and Y.-H. Ahn, Bull. Korean Chem. Soc. 31, 1067 (2010).

${ }^{25}$ Y. Wang, W. Wang, L. Zhong, J. Wang, Q. Jiang, and X. Guo, Appl. Surf. Sci. 256, 3837 (2010).

${ }^{26}$ Y. Liu, X. Yin, J. Zhang, Y. Wang, Z. Han, and L. Ren, Appl. Surf. Sci. 280, 845 (2013).

${ }^{27}$ A. G. Demir, B. Previtali, and C. A. Biffi, Adv. Mater. Sci. Eng. 2013, 692635 (2013).

${ }^{28}$ Y. Jee, M. F. Becker, and R. M. Walser, J. Opt. Soc. Am. B 5, 648 (1988),

${ }^{29}$ H. P. Jennissen, Mat.-wiss. u. Werkstofftech. 42, 1111 (2011).

${ }^{30}$ S. Housh, B. Mikucki, and A. Stevenso, ASM Handbook, Online edition (American Society for Metals, Metals Park, 2014), http://products.asmin ternational.org/hbk/index.jsp.

${ }^{31}$ J. Hoffman, Z. Szymański, and V. Azharonok, AIP Conf. Proc. 812, 469 (2006). 